\title{
Two Phase Change Material with Different Closed Shape Fins in Building Integrated Photovoltaic System Temperature Regulation
}

\author{
M. J. Huang ${ }^{1, *}$ \\ ${ }^{1}$ University of Ulster, Newtownabbey, Co. Antrim, N. Ireland, UK \\ * Corresponding author. Tel: +44 28 90366037, E-mail: m.huang@ulster.ac.uk
}

\begin{abstract}
Photovoltaics (PVs) operate at around $40^{\circ} \mathrm{C}$ above ambient temperature in full sun. On a cold day in Europe the cell temperature will be at $30^{\circ} \mathrm{C}$ and compared to a summer temperature of up to $80^{\circ} \mathrm{C}$. As each ten temperature increases the efficiency of the crystal silicon photovoltaic will reduce $10 \%$. So considering the whole year, running at $25^{\circ} \mathrm{C}$ for Building Integrated Photovoltaic (BIPV) will be an ideal temperature target to achieve in order to keep PV cells at their peak efficiency in Europe. Passive heat removal technique was applied for thermal regulation of PV using Phase Change Material (PCM) integrated on the back of the PV. The temperature in PV can be effective regulated, but the low thermal conductivity of the PCMs is one of the main problems for this application. This paper details the results of a theoretical investigation and analysis of PV temperature control and solar thermal energy storage achieved using phase change materials with different types of fins, structure and PCMs. The predicted performance provides an insight into the effects of using various quantities of different PCM materials with different types of fins and thermal storage for selected ambient conditions of temperature and insolation. From this parametric study, optimum arrangements of the PV/PCM system with different type of fins are proposed, thereby improving the efficiency of the PV/PCM system.
\end{abstract}

Keywords: phase change materials, Photovoltaic and Building Integrated Photovoltaics

\section{Nomenclature}

C Specific heat capacity

E Thermal energy

$H \quad$ Heat transfer coefficient $\quad W^{-2} K^{-1}$

$H \quad$ Latent thermal energy.......................... $\mathrm{Jkg}^{-1}$

$I_{T} \quad$ Insolation incident on photovoltaic cell $W m^{-2}$

K Thermal conductivity ................... $\mathrm{Wm}^{-1} \mathrm{~K}^{-1}$

$T$ Time

$\Delta t$ Time step.

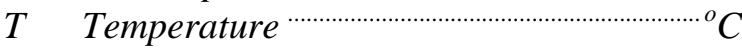

$\Delta T$ Transition temperature of PCM ${ }^{\circ} \mathrm{C}$

$T_{P V} \quad P V$ Temperature

\section{Introduction}

Building integrated photovoltaic systems (BIPVs) are widely recognised as the most cost effective form of PV power generation [1]. As well as producing electricity, BIPV panels can replace some of the conventional wall cladding and roofing materials, therefore reducing the net costs of the PV system. The elevation of the PV temperature reduces solar to electrical energy conversion efficiency by $0.4-0.5 \% \mathrm{~K}^{-1}$ for crystal silicon PV when it rises above the characteristic power conversion temperature of $25^{\circ} \mathrm{C}$ [2] [3]. Maintaining the silicon PV's temperature at a low temperature, preferably lower than or around $25^{\circ} \mathrm{C}$ will retain the maximum conversion efficiency of the PV for practical applications. Active and passive heat dissipations have been studied for decade [4] [5] [6].

PCM can absorb a large amount of energy during the phase change, and is therefore widely investigated for thermal storage. A review on thermal energy storage with phase change materials and application has been carried by Sharma, et al. [7] and Agyemin et. al. [8]. An 
investigation of a system which uses PCMs to absorb energy as latent heat at a constant phase transient temperature and to regulate the rise in PV temperature (PV/PCM) has been carried out recently ([6], [9] and [10]). It was found that the PCM thermal conductivity and volume expansion during melting are the main barriers for this application. A series of arrangements with different types of fins inserted inside the PV/PCM system was also carried out experimentally [6]. Although the metal fins inserted inside the PCM can improve the heat transfer inside the PV/PCM system, the thermal regulation period declines as the volume of the PCM is substituted by the metal mass of the PV/PCM system. It was also found that during crystallisation the air cavity formed inside the PCM will increase the thermal resistance when used for temperature regulation during the day time. The further studies using different types of PCMs for the PV/PCM application, including a eutectic mixture of capriclauric acid (CL), a commercial blend of salt hydrate and paraffin phase change material (SP22), a eutectic mixture of Capric Palmitic acid (CP) and a Calcium Chloride hexa hydrate $\left(\mathrm{CaCl}_{2}\right)$, have been carried out [11]. Eutectic Mixture of Capric-Palmitic Acid and $\mathrm{CaCl} 2.6 \mathrm{H} 2 \mathrm{O}$ with higher thermal conductivities than paraffin wax have a better thermal regulation performance on BIPV for indoor conditions and the outdoor climates of Ireland and Pakistan [11]. Further investigation on the corrosion of the container needs to be conducted.

In this paper an experimental validated numerical simulation model [9] has been modified to suit for two phase change materials for PV/PCM modeling. The thermal regulation of the PV/PCM system in triangular shaped cells and circular shaped cells (which optimise for reducing stress due to PCM expansion) have been studied. A range of different phase transient temperature PCMs under static state and realistic conditions have been discussed in this paper.

\section{Methodology}

The simulation model used in this work is a two dimensional temperature-based finite volume based conjugated heat transfer numerical model to moderate the temperature rise in BIPVs in a PV/PCM system. This model is based on the previously developed and experimentally validated model for a single PCM with straight fins in the PV/PCM system by the authors [9]. The non-linear transient model uses Boussinesq's approximation and allows convection and diffusion to be simulated. The developed model can be used to predict the transient temperature distribution and fluid flow field within a two-dimensional region in the PV/PCM system for different insolation, ambient temperatures, convective and radiative heat transfer boundary conditions ([9] and [10]). The modified PV/PCM model can be used for multiple PCMs with different transient temperatures and for triangular shaped PCM cells. The following assumptions are made:

(i) The heat conduction in the PV/PCM combined system is two-dimensional and the end sides at the top and bottom are adiabatic.

(ii) The thermal conductivity of the aluminum frame and PCMs in the solid and liquid phases are constant and do not vary with respect to temperature.

(iii) The PCM is homogeneous and isotropic.

(iv) The convection effect in the molten PCM is neglected for the thermal performance comparison, but has been considered for special case.

(v) The interfacial resistances are negligible.

(vi) The specific heat capacity " $C_{P}$ " value of the PCM is considered as uniform during phase change process, though in actual practice, there is variation in $\mathrm{C}_{\mathrm{P}}$ value within the small temperature range. 
A brief summarization is as follows.

The energy equation for melt [9]:

$$
\rho_{L} c_{L} \frac{\partial T}{\partial x}+\frac{\partial}{\partial x}\left(\rho_{L} c_{L} \vec{u} T-k_{L} \frac{\partial T}{\partial x}\right)+\frac{\partial}{\partial y}\left(\rho_{L} c_{L} \vec{v} T-k_{L} \frac{\partial T}{\partial y}\right)=0
$$

The energy equation for solid:

$$
\rho_{S} c_{S} \frac{\partial T}{\partial t}+\nabla \cdot\left(k_{S} \nabla T\right)=0
$$

Where the same equations hold good for all the frame and cell wall material and PCMs by incorporating suitable $k, \rho, C_{p}$ values. The instantaneous continuity of heat flux and temperature at the interfaces of frame and cell with PCMs are preserved.

In the exterior front boundary, where the PV/PCM system is exposed to solar radiation, the boundary condition is,

$$
\left.k \frac{\partial T}{\partial x}\right|_{x=0}=S+h_{0}\left(T_{a m b}-T_{x=0}\right)
$$

In the exterior back layer of the PV/PCM system $x=L$, the boundary condition is

$$
\left.k \frac{\partial T}{\partial x}\right|_{x=L}=h_{L}\left(T_{x=L}-T_{a m b}\right)
$$

Where $h_{0}$ and $h_{L}$ are the heat transfer coefficients from the front and back surfaces of the PV/PCM system to the surroundings. $S$ is the heat received by the PV/PCM system on the front surface from the incident solar energy.

Table 1. Thermophysical properties of RT21, RT27, RT31 [13]

\begin{tabular}{|l|l|l|l|l|l|}
\hline \multicolumn{2}{|l|}{} & RT21 & RT27 & RT31 & Aluminium \\
\hline Melting temperature & 21 & 27 & 29 & N/A \\
\hline Latent heat $\left(\mathrm{kJ} \mathrm{kg}^{-1}\right)$ & 134 & 184 & 169 & N/A \\
\hline Density $\left(\mathrm{kg} \mathrm{m}^{-3}\right)$ & & & & \\
Liquid & 760 & 750 & 770 & N/A \\
Solid & 840 & 840 & 890 & 2675 \\
\hline Thermal conductivity $\left(\mathrm{Wm}^{-1} \mathrm{~K}^{-1}\right)$ & 0.2 & 0.2 & 0.2 & 160 \\
\cline { 2 - 6 } & Viscosity $\left(\mathrm{mm}^{2} \mathrm{~s}^{-1}\right)$ & 25.71 & 26.32 & 28.57 & N/A \\
\hline
\end{tabular}

The most significant thermal characteristics affecting the performance of the PV/PCM system are (a) the PCM heat capacity, (b) the phase transient temperature, (c) the location of the PCM and (d) the mass in the system fins arrangement. In this paper, the PV/PCM system has been designed with small metal cells to hold two types of PCMs considered to enhance heat transfer. The triangular and circular cell shapes are good for directing the bubbles produced during the melting process and thereby dissipates the stress due to the volume expansion which challenges many PCM applications. The schematic of the PV/PCM with metal cells is illustrated in Figure 1. The vertical position is used to mimic building integrated PV. The study here is just concentrated on the building wall integrated PV, the inclined PV systems beyond these limits. The $4 \mathrm{~mm}$ aluminium front/ back walls and the $1 \mathrm{~mm}$ aluminium alloy fins of the PV/PCM test system provided a high rate of heat transfer to the PCM. The interior dimensions of the containers were $0.132 \mathrm{~m}$ high by $0.04 \mathrm{~m}$ depth. The upper and lower ends of the PV/PCM system were assumed adiabatic. The incident energy $I_{T}$ absorbed by the PV as heat is conducted through the high heat transfer cell wall to the PCM and dissipated from the rear of the PV/PCM system. The different thermal regulation characters of the PCMs can hold PV temperature at lower levels for longer periods. The PCMs that are commercially available 
with different phase transient temperatures from 21 to $60^{\circ} \mathrm{C}$ are combined to regulate the PV temperature rising in the PV/PCM system. Different combinations of the PCMs used to augment the PV/PCM system are analysed for static conditions and realistic diurnal temperature and insolation boundary conditions in the England summer period and the heat transfer and temperature distribution are predicted. The thermal properties of the aluminium alloy and the four PCMs that can be combined into the types of PCMs from RUBITHERM [13] used as input data in the simulations are presented in Table 1.
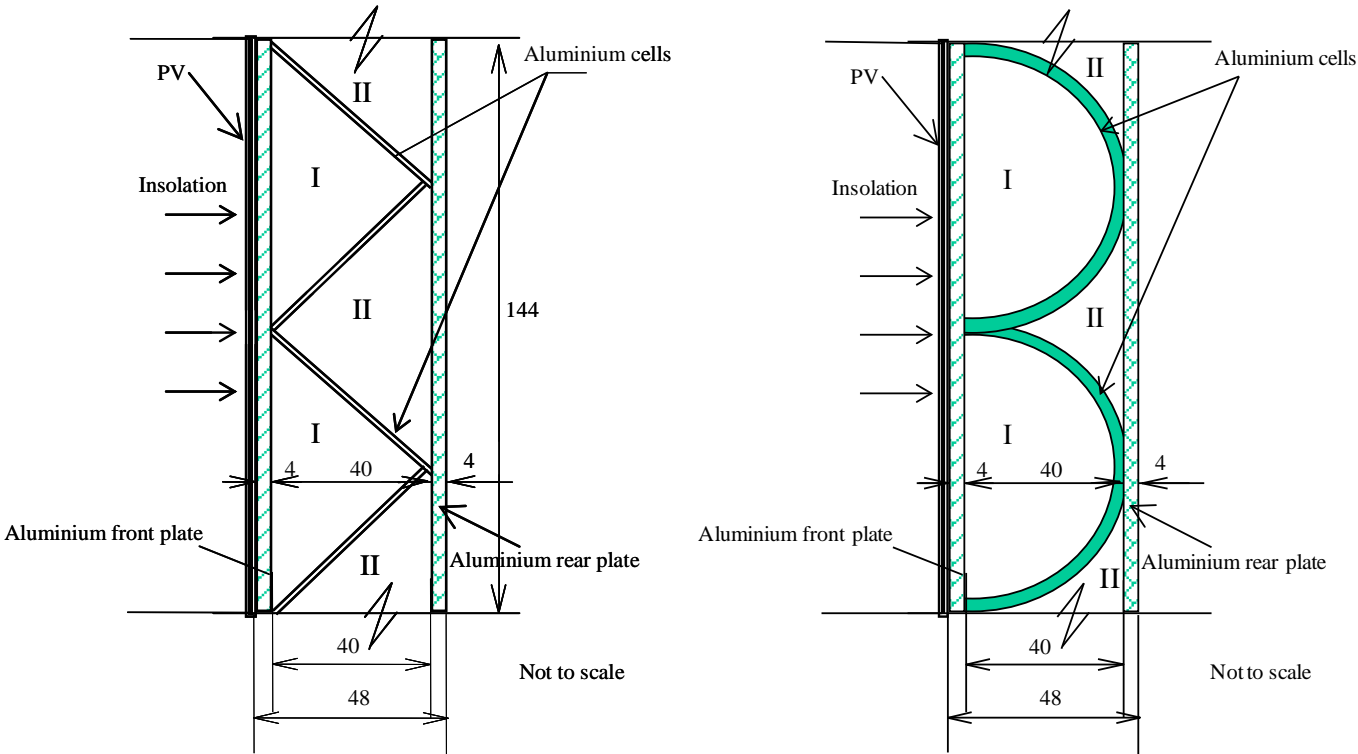

Figure 1. Schematic diagram of PV/PCM system with metal cells for different PCMs
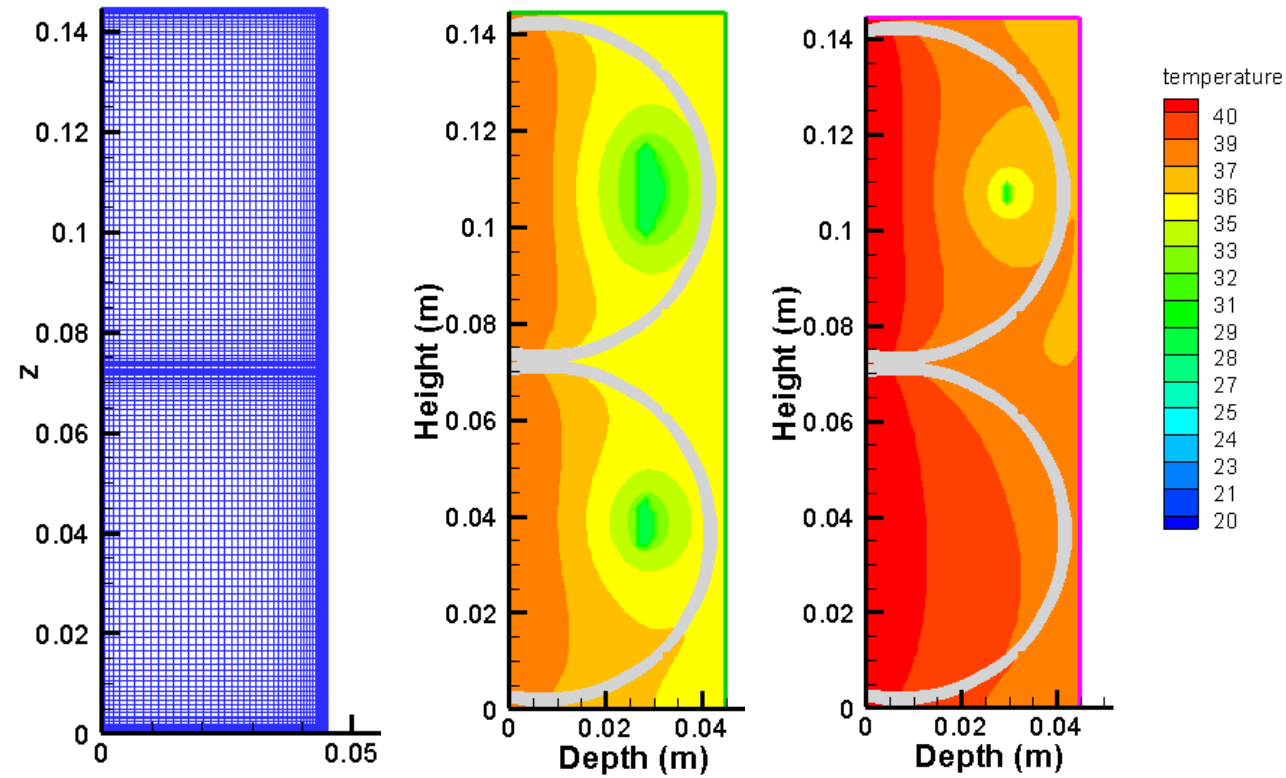

Figure 2. Grid and isotherms of circular cells with two phase change materials in PV/PCM system

The heat transfer coefficients from the front and back surfaces of the PV/PCM system are set at $10 \mathrm{Wm}^{-2} \mathrm{~K}^{-1}$ and $5 \mathrm{Wm}^{-2} \mathrm{~K}^{-1}$ (to simulate natural ventilation conditions) and the top and bottom boundaries of the system are assumed to be adiabatic. A fixed grid space of $1 \mathrm{~mm}$ square for straight fins and variable grid for circular cell fins simulation with finite volumes and a variable time step with a minimum value of $0.0125 \mathrm{~s}$ are used for all the simulations. The total number of grid is $144 \times 48$ for simulation. 
Simulating the behaviour of the PV/PCM system with the two different phase transient temperature PCMs is carried out under the static state and realistic conditions. In real thermal applications it is subject to the cyclic melting and solidification boundary conditions. To predict long-term temperature control, three days are simulated using weather data for the $21^{\text {st }}$ June for the SE of England [14] on the vertical south-east oriented PV/PCM system when the insolation was greater than $120 \mathrm{Wm}^{-2}$. For the simulations, realistic ambient temperatures and insolation boundary conditions are regarded as invariant over 5 minute intervals. The simulation temperatures within the PV/PCM system are all initially set to the outdoor ambient temperature at 00:00 $\mathrm{hr}$ for the transient applied boundary condition.

\section{Results and Discussions}

In order to evaluate the PV/PCM performance, predictions of the temperature development with the two phase materials, a single PV plate is predicted as a reference for performance comparison. The predicted reference temperature is at $68.45^{\circ} \mathrm{C}$ with insolation $1000 \mathrm{~W} / \mathrm{m}^{2}$ and ambient temperature $20^{\circ} \mathrm{C}$. The following four cases using different combinations of two PCMs with different melting temperatures and two shapes of fins (triangle and half circular fins) were simulated on setting static conditions of (a) insolation $1000 \mathrm{~W} / \mathrm{m}^{2}$ and ambient temperature $20^{\circ} \mathrm{C}$ and (b) realistic three days ambient conditions with repeating data on $1^{\text {st }}$ June [14].

- $\quad$ RT27 with RT21 (triangle cells)

- RT27 with RT27 (triangle cells)

- RT31 with RT27 (triangle cells)

- RT31 with RT27 (half circular cells)

- RT60 with RT21 (triangle cells)

The variable grids of the simulation for the circular cell is listed in Figure 2 along with the isotherms of the circular cells in different time. The insolation absorbed on the front surface of the PV/PCM system and conducted through the PV increases the temperature of the metal cell wall. The metal wall of the cell provides good thermal transfer to the two PCMs. Similar situations can be seen on the triangle fins in Figure 3. As time elapses, it can be seen that the temperature on the front surface of the PV/PCM system has a lower temperature rise for the system with RT27-RT21 than that with the RT27-RT27 and RT31-RT27 filled system, and the thermal regulation period is shorter. It is easy to understand that the lower melting PCM can have better thermal regulation compared with the higher melting PCM. This can be observed from the predicted isotherms for the PV/PCM system cross-sections as shown in Figure 3. Metal fins in the PCM increase the heat transfer inside of the PCMs by increasing the surface area over which heat transfer to the PCM occurs and also act as a pressure release pathway for the melted PCM. After the first 30 minutes the temperature inside the system with RT27-RT21 is relatively lower than the RT27-RT27 and RT31-RT27 cases. After 60 minutes the front temperature on RT27-RT21 increases more rapidly to the insolation intensity than the RT27-RT27 and RT31-RT27 cases do, and the thermal regulation period is less. The position of the PCMs is an important factor in thermal regulation. When combined with low phase change transient PCM the temperature on the front surface of the PV/PCM system has a lower temperature rise. 


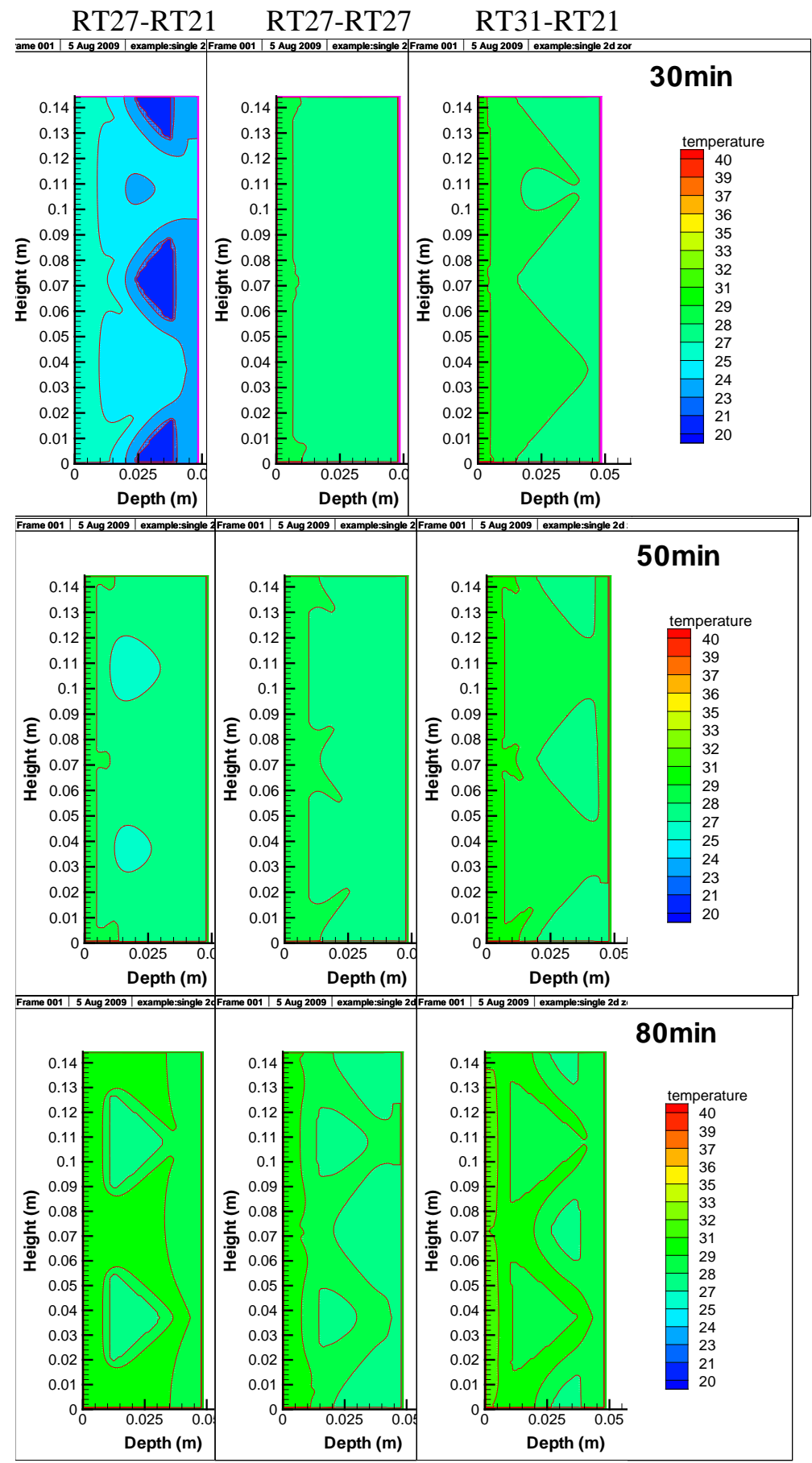

Figure 3. Predicted isotherms for the PV/PCM system with four cases of RT27-RT21, RT31-RT27 during the PCM phase change process

A three day simulation using the weather data on $21^{\text {st }}$ of June has been undertaken to predict the heat accumulation in the PV/PCM system with a combination of two PCMs and is presented in Figure 4. The temperatures of the system respond more rapidly to the insolation than to the ambient temperatures. When no insolation is involved, it can be found that the temperature on the front surface of the system decreases with phase change properties. The temperature of the PV/PCM systems follows the incident insolation but lags with the properties of PCMs by more than 20 minutes. Absorbed solar energy is stored in the PCMs during high daytime temperatures and subsequently released to the ambient in the evening. The predicted temperatures for the second and third days for all the cases are the same, the 
PCM has thus released all its latent heat to the ambient environment at night and returned to its solid phase at the start of each period of insolation. The RT31-RT27 system with triangle and circulate close fins can efficiently control the temperature on the PV under $30^{\circ} \mathrm{C}$ for the whole test period. For two different PCMs the lower phase transient PCM dominates the whole system performance. The phase transient performance is clear in the cooling stage.

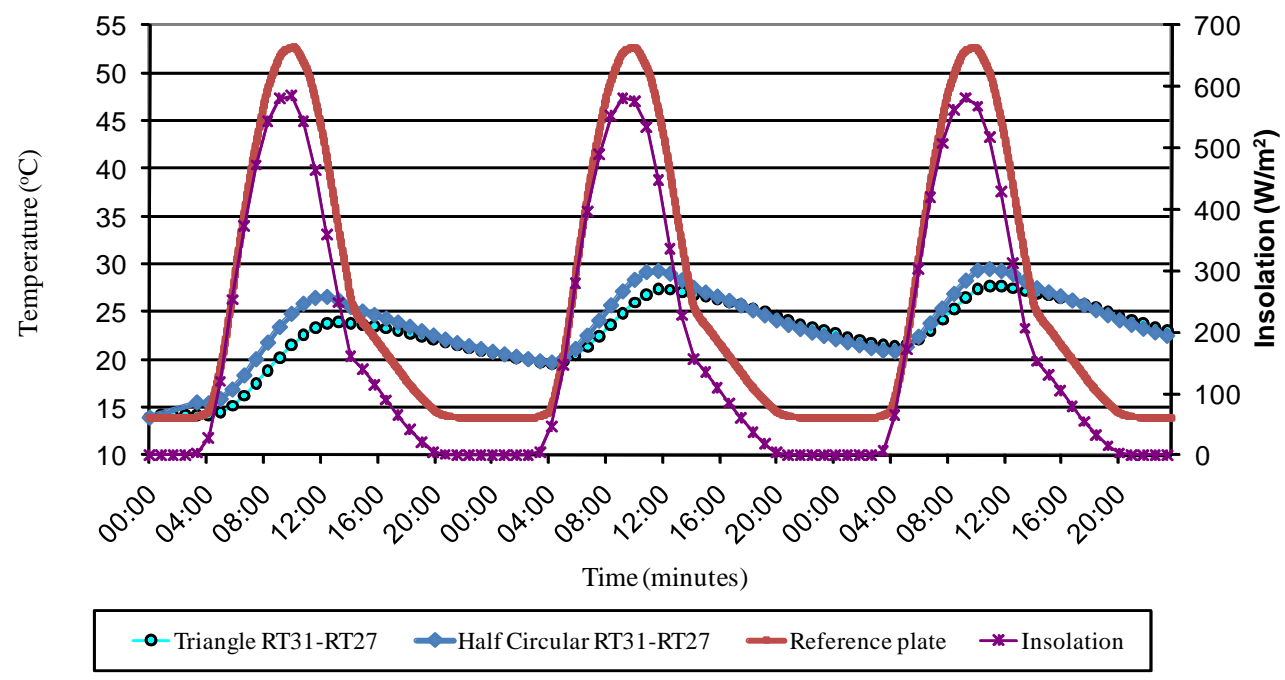

Figure 4 Average temperature evolution at the front surface of the PV/PCM system with the RT31 and RT27 combination for a three day simulation using the weather data on $21^{\text {st }}$ of June SE England

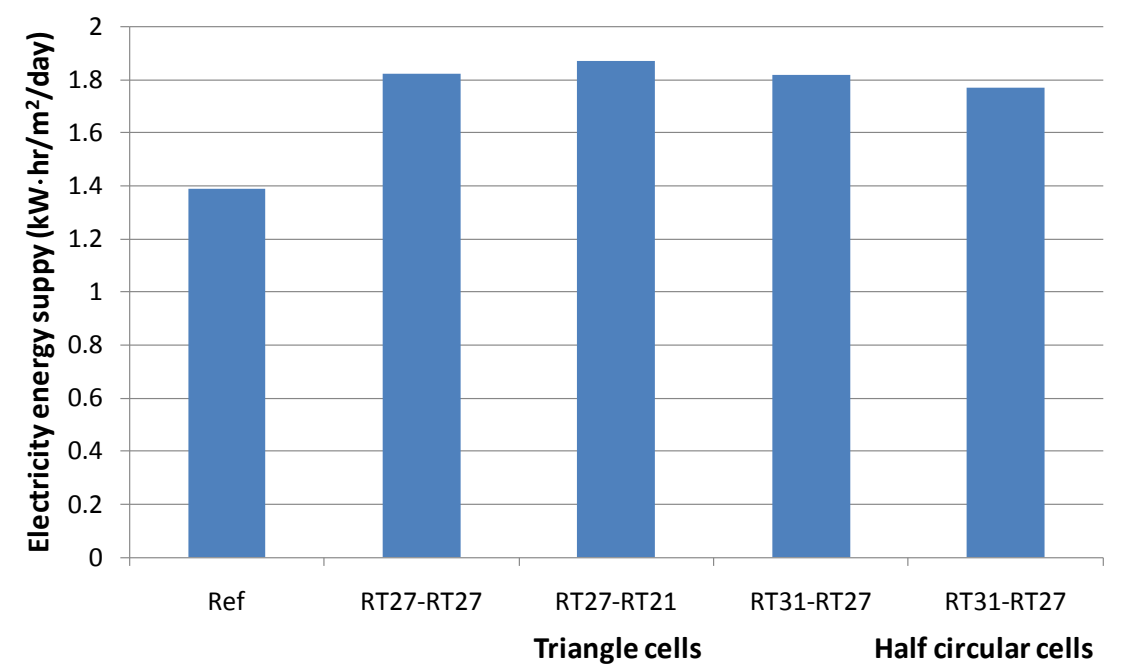

Figure 5 Electricity energy supply on the selected day in 23th June in SE England per square metre for different PCMs combine PV/PCM system along with reference PV module

The temperature regulation may be quantified in terms of the electricity supplied in a typical day in summer. Assuming that the useful electricity power can be produced only after the insolation is over $200 \mathrm{Wm}^{-2}$. Figure 5 shows the comparison of the electricity energy provided by the two PCMs combined PV/PCM systems with the reference PV module during one day's performance in $21^{\text {st }}$ June in SE England. The PV/PCM systems with half circular cells and triangle cells have been compared as well. The effect of temperature regulation by PV/PCM combined system is clear. The difference between using the triangle and half circular cells is not significant with the selected PCMs combination. 


\section{Conclusions}

The thermal performance of using two PCMs to regulate the temperature rising on PV/PCM system is studied. The PCMs with different transient temperatures can maintain the PV at operating temperature closer to its characteristic value of $25^{\circ} \mathrm{C}$ and thus lead to an improvement in solar-to-electrical conversion efficiency. The different thermal regulation characters of the PCMs can keep PV temperature at lower levels for longer periods. The PCMs evaluated at different combinations show that the thermal regulation performance of the PV/PCM depends on (a) the thermal mass of PCMs, (b) the positions of the PCMs inside the PV/PCM system and (c) the thermal characteristics of both the PCMs and the PV/PCM systems structure. Comparing different combinations, RT27-RT21 achieves the highest temperature reduction during the daily operation.

\section{References}

[1] NREL, (2008). National Renewable Energy Laboratory, A National Laboratory of the US Department of Energy Office of Energy Efficiency \& Renewable Energy, http://www.nrel.gov/pv/building_integrated_pv.html.

[2] Ingersoll J. G., (1986). Simplified calculation of solar cell temperatures in terrestrial photovoltaic arrays. ASME J. Solar Energy Engineering 108, 95-101

[3] Krauter S., Hanitsch R. and Wenham S. R., (1994). Simulation of thermal and optical performance of PV modules, Renewable Energy, 5, 3, 1701-1703

[4] Tonui, J.K. and Tripanagnostopoulos, (2007). Air cooled PV/T solar collectors with low cost performance improvements. Solar Energy 81, 498-511.

[5] Fossa, M., Ménézo, C., Leonardi, E., (2008). Experimental natural convection on vertical surfaces for building integrated photovoltaic (BIPV) applications, Experimental Thermal and Fluid Science 32, 980-990.

[6] Huang M.J., Eames P.C. and Norton B., (2006). Experimental Performance of Phase Change Materials for Limiting Temperature Rise Building Integrated Photovoltaics, Journal of Solar Energy, 80, pp. 1121-1130.

[7] Sharma, A., Tyagi, V. V., Chen, C. R. and Buddhi, D. (2009). "Review on thermal energy storage with phase change materials and applications." Renewable and Sustainable Energy Reviews 13(2): 318-345.

[8] Agyenim, F., Hewitt, N., Eames, P. and Smyth, M. (2010). "A review of materials, heat transfer and phase change problem formulation for latent heat thermal energy storage systems (LHTESS)." Renewable and Sustainable Energy Reviews 14(2): 615-628.

[9] Huang M.J., Eames P.C. and Norton B., (2004). Thermal Regulation of BuildingIntegrated Photovoltaics Using Phase Change Materials, International Journal of Heat and Mass Transfer, 47, Pages 2715-2733

[10]Huang M.J., The Application of CFD to Predict the Thermal Performance of Phase Change Materials for the Control of Photovoltaic Cell Temperature in Buildings, PhD Thesis, University of Ulster, UK, 2002.

[11] Hasan, A. (2010), Phase Change Materials for Thermal Regulation of Building Integrated Photovoltaics, PhD thesis, Dublin Institute of Technology.

[12] Duffie J.A. and Beckman W.A., (1991). Solar Engineering of Thermal Processes, Wiley \& Sons, Inc., USA.

[13]Anon, RUBITHERM data sheets for RT21, RT27, RT31 and RT60, RUBITHERM GmbH, Schumann company. (2011).

[14]CIBSE, (2006). CIBSE Guides A, Chartered Institute of Building Services Engineers, London, UK. 